\title{
The COVID-19 Ibuprofen controversy; a systematic review and meta-analysis of NSAIDs in adult acute lower respiratory tract infections
}

\author{
Ricky Vaja ${ }^{1}$, Jeffrey Chan ${ }^{2}$, Plinio Ferreira ${ }^{1}$, Amer Harky $^{3}$, Luke Rogers ${ }^{4}$, Hime Gashaw ${ }^{1}$, \\ Nicholas Kirkby ${ }^{5}$, and Jane Mitchell ${ }^{5}$ \\ ${ }^{1}$ Imperial College London \\ ${ }^{2}$ Prince of Wales Hospital \\ ${ }^{3}$ Liverpool Heart and Chest Hospital NHS Foundation Trust \\ ${ }^{4}$ University Hospitals Plymouth NHS Trust \\ ${ }^{5}$ Imperial College of London
}

June 5, 2020

\begin{abstract}
In light of the recent safety concerns relating to NSAID use in COVID-19, we sought to evaluate cardiovascular and respiratory complications in patients taking NSAIDs during acute lower respiratory tract infections. We carried out a systematic review and meta-analysis of randomised controlled trials and observational studies. Studies of adult patients with short-term NSAID use during acute lower respiratory tract infections, including bacterial and viral infections, were included. Primary outcome was allcause mortality. Secondary outcomes were cardiovascular, renal and respiratory complications. In total, eight studies including two randomised controlled trials, three retrospective and three prospective observational studies enrolling 44140 patients were included. Five of the studies were in patients with pneumonia, two in patients with Influenza, and one in patients with acute bronchitis. There was uncertainty as to the effects on mortality (RR $0.87[0.63,1.18])$, but pleuro-pulmonary complications were more common with NSAID use (RR $2.62[1.96,3.50])$. However, all studies exhibited high risks of bias, primarily due to confounding variables. Cardiovascular outcomes were not reported by any of the included studies. Short-term NSAID use during acute lower respiratory tract infections was associated with more pleuro-pulmonary complications although this may be due to confounding by indication. There remains significant uncertainty on the effects on mortality. Such results should be interpreted cautiously given the very low quality of evidence. Mechanistic and clinical studies addressing the captioned subject are urgently needed, especially in relation to COVID-19.
\end{abstract}

\section{Hosted file}

Manuscript BJP Final.doc available at https://authorea.com/users/330131/articles/456985-thecovid-19-ibuprofen-controversy-a-systematic-review-and-meta-analysis-of-nsaids-in-adultacute-lower-respiratory-tract-infections 